\title{
ETV6-NTRK3 and STRN-ALK kinase fusions are recurrent events in papillary thyroid cancer of adult population
}

\author{
André Uchimura Bastos, Ana Carolina de Jesus and Janete Maria Cerutti \\ Departamento de Morfologia e Genética, Genetic Bases of Thyroid Tumors Laboratory, Disciplina de Genética, \\ Universidade Federal de São Paulo, São Paulo, Brazil
}

\author{
Correspondence \\ should be addressed \\ to J M Cerutti \\ Email \\ j.cerutti@unifesp.br
}

\begin{abstract}
Objective: PTC-specific analysis identified novel fusions involving RET, BRAF, NTRK1, NTRK3, AGK and ALK genes in adults and pediatric PTCs. Although many novel fusions are PTC-specific events and, therefore, are ideal for diagnosis purposes, validation across additional and larger patient cohorts is essential for introducing these potential diagnostic or prognostic biomarkers into the clinical practice. As most of the $B R A F, N T R K 3$ and $A L K$ fusions were initially found in pediatric PTC or in more aggressive thyroid carcinomas, and there is a great disparity across population, in this study, we screened a large set of adult-sporadic PTC cases for the most prevalent kinase fusion lately described in the TCGA. Design and methods: The prevalence of the fusions was determined by RT-PCR in 71 classical PTC, 45 follicular variants of PTC (FVPTC), 19 follicular thyroid adenomas (FTAs) and 22 follicular thyroid carcinomas (FTCS).

Results: ETV6-NTRK3 was exclusively found in FVPTC, in both encapsulated and infiltrative variants, but was not found in FTAs and FTCs. STRN-ALK was found in both classical PTC and FVPTC. No AGK-BRAF fusion was identified in this series, endorsing that $A G K-B R A F$ is a genetic event mainly associated with pediatric PTCs.

Conclusions: The identification of kinase fusions in thyroid carcinomas helps to expand our knowledge about the landscape of oncogenic alterations in PTC. As ETV6-NTRK3 and STRN-ALK are recurrent and not identified in benign lesions, they can certainly help with diagnosis of thyroid nodules. Further analysis is needed to define if they can also be useful for prognosis and guiding therapy.
\end{abstract}

\section{Introduction}

Chromosomal rearrangements, which cause the production of a functional fusion gene, were the first mechanism of oncogene activation discovered in human cancers in 1973, particularly in hematologic malignancies $(1,2)$. The first gene fusion found in malignant epithelial tumors was reported in 1987, when the DNA isolated from a primary papillary thyroid carcinoma (PTC) was successfully delivered to NIH3T3 cells through transfection (3). Cytogenetic and molecular findings demonstrated that the gene fusion occurred due to a paracentric inversion of chromosome 10, which juxtaposes the intracellular tyrosine kinase domain of the RET to the $5^{\prime}$ sequence of
() 2018 European Society of Endocrinology Printed in Great Britain the CCDC6, which is expressed in thyroid follicular cells $(3,4,5)$. This novel fusion transcript was named RET/PTC, as it encompasses the RET gene and was exclusively found in PTC (3).

The following decade was characterized by the discovery of several novel fusions involving RET gene in both sporadic and radiation-induced PTCs. RET/PTC represents the major group of gene fusions found in PTC, with RET/PTC1 and RET/PTC3 being the most prevalent isoforms (reviewed in $(6,7,8)$ ). Importantly, in vitro and in vivo analyses confirmed that RET/PTC fusions have a critical role in thyroid carcinogenesis $(9,10,11)$. 
Recently, advances in genomic technologies have uncovered novel fusion genes in thyroid tumors. The Cancer Genome Atlas (TCGA) Pan-Cancer project has generated comprehensive multidimensional maps of the key genomic changes in 33 tumors types, including PTC. PTC-specific analysis not only confirmed the significance of known fusions (RET and NTRK1) but also identified novel fusions involving kinases that very likely play a role in thyroid cancer such as BRAF, NTRK3 and ALK (12).

Although thyroid cancer showed lower frequency of kinase fusions compared to other tumor types, a high percentage of these events were recurrent, confirming their role in the pathogenesis of thyroid carcinomas. Among the novel gene fusions, some isoforms involving $B R A F$, NTRK3 and ALK genes were acknowledged to play a role in the pathogenesis of pediatric PTC or more aggressive variants of PTC.

Regarding fusions involving the BRAF gene, $A G K-B R A F$ was primarily described in post-Chernobyl radiation-induced thyroid tumors (13) and later identified as a recurrent event in sporadic pediatric PTC (14). Moreover, among the isoforms identified, $A G K-B R A F$ was functionally characterized and showed to be able to induce MAPK activation and to increase cell proliferation and transformation in NIH3T3 or COS7 transfected cells (13).

ETV6-NTRK3 is the most common rearrangement found after any RET/PTC isoform in the TCGA (12). While its prevalence was very low (1\%) in PTC from adults (12, $15)$, it is the second most common rearrangement in radiation-exposed PTC $(13,15)$. Additionally, ETV6-NTRK3 has been shown to be able to induce MAPK activation and increase cell proliferation and transformation (13). Although other novel isoforms of NTRK3 fusions were described, only one sample was found to be positive for the NTRK3 fusion and the transforming potentials of these fusion proteins were not determined.

Finally, concerning $A L K$ fusions, STRN-ALK was found as a very rare event identified in more aggressive variants of thyroid cancer (16) and in conventional PTCs (17). Importantly, its ectopic expression was able to induce MAPK activation, increase cell proliferation and transformation and induce tumor formation in xenograft models (16). Although novel ALK fusion variants were described in PTC, the isoforms were not found to be recurrent or were not functionally characterized.

Unlike RET/PTC, these fusions were not found in adjacent normal thyroid tissues or benign neoplasms $(12,13,16,17)$, which support the pathogenic role of these fusions in PTC.
As genetic and environmental variations contribute to population-based disparities and we enter the era of precision medicine and molecular diagnosis, we questioned about the implications of these novel fusions in the diagnosis of PTC from different geographical areas. Additionally, validation analysis in a large and independent set of malignant and benign lesions is indubitably essential to better define the relevance of these novel fusions in the pathogenesis of thyroid tumors and, consequently, in the differential diagnosis of thyroid nodules.

We here reported the prevalence of ETV6-NTRK3, $S T R N-A L K$ and $A G K-B R A F$ fusions in a large Brazilian cohort of adult-sporadic PTC. We additionally assessed the prevalence of these fusions in other benign and malignant thyroid tumors.

\section{Methods}

\section{Sample selection}

The series consists of primary tumors from patients who underwent thyroid surgery from 2000 to 2007 at Hospital São Paulo, Universidade Federal de São Paulo and Hospital das Clínicas, Faculdade de Medicina da Universidade de São Paulo. The study included 116 PTCs, 71 (61\%) classical PTCs and 45 (39\%) follicular variants of papillary thyroid carcinoma (FVPTC). The final histological classification on surgical specimens was obtained from formalin-fixed paraffin-embedded sections. The FVPTC is defined as a tumor composed entirely or almost entirely of cells with a follicular architecture exhibiting nuclear features of classical PTC. The FVPTC can be completely encapsulated (EFVPTC) or partially encapsulated or infiltrative (IFVPTC). Additionally, 19 follicular thyroid adenomas (FTAs) and 22 follicular thyroid carcinomas (FTCs) were used to investigate the presence of ETV6-NTRK3, STRN-ALK and $A G K-B R A F$ in other benign and malignant thyroid tumor subtypes. None of the patients had a history of previous radiation exposure. Informed consent was obtained from all subjects involved in this study. The study was conducted under the approval of the Review Boards and Research Ethical Committees of the affiliated institutions. The demographic and clinico-pathological features such as the age at onset, gender, tumor size, histological variant, multifocality, extrathyroidal invasion, the presence of lymph node metastasis and recurrence were correlated with the genetic profile. 


\section{Detection of fusion transcripts by standard RT-PCR}

All the samples were screened for the presence of ETV6NTRK3, STRN-ALK and AGK-BRAF by standard RT-PCR. For the detection of fusion transcripts, primers were designed within exons located near the breakpoint regions described in PTC (Fig. 1 and Table 1).

Total RNA was isolated from the core of the tumor (in an attempt to avoid contamination with normal tissue) using Trizol (Invitrogen, Thermo Fisher Scientific), according to the manufacturer's instructions. Total RNA concentration and purity were analyzed using a Nanodrop ND-2000 spectrophotometer (Thermo Fisher Scientific). RNA integrity was evaluated using agarose gel electrophoresis or Agilent 2100 Bioanalyzer System (Agilent Technologies). Total RNA $(1 \mu \mathrm{g})$ was treated with DNAse and reverse-transcribed into cDNA with oligo-dT $\mathrm{T}_{12-18}(0.5 \mu \mathrm{g})$ using a Superscript III reverse transcriptase kit (Invitrogen) as described (18). An aliquot of cDNA $(1 \mu \mathrm{L})$ was subjected to PCR amplification using $10 \mathrm{mM}$ Tris- $\mathrm{HCl}$ (pH 8.4), $50 \mathrm{mM} \mathrm{KCl}, 1.5 \mathrm{mM} \mathrm{MgCl}_{2}$, $200 \mu \mathrm{M}$ of each dNTP, 1 unit of Platinum Taq DNA polymerase (Invitrogen) and $200 \mathrm{nM}$ of each specific primer for the target genes or reference gene (RPS8). PCR reaction was performed as follows: initial denaturation at $95^{\circ} \mathrm{C}$ for $5 \mathrm{~min}$ followed by 40 cycles of $95^{\circ} \mathrm{C}$ for $30 \mathrm{~s}$, annealing temperature for $30 \mathrm{~s}$ and $72^{\circ} \mathrm{C}$ for $30 \mathrm{~s}$, followed by a final extension of $72^{\circ} \mathrm{C}$ for $2 \mathrm{~min}$. The PCR products were analyzed by electrophoresis on a $2 \%$ agarose gel and visualized on a Bio-Rad Gel Doc EZ system (Bio-Rad Laboratories). The presence of the fusion transcripts was confirmed by direct sequencing of PCR products using the BigDye Terminator Cycle Sequencing Kit (Thermo Fisher Scientific) as previously described (19). The primers used to detect fusions, reference gene, annealing temperatures and expected PCR product sizes are detailed in Table 1.

\section{Expression of fusion oncogenes in PCCL3 cell line}

PCCL3 (normal follicular thyroid cells derived from Fischer rats) were cultured in Ham's F12 medium supplemented with 5\% FBS (Gibco, Thermo Fisher Scientific) and 4 hormone-mixture including thyrotropin $(1 \mathrm{U} / \mathrm{mL})$, hydrocortisone $(10 \mathrm{nM} / \mathrm{mL})$, transferrin $(5 \mu \mathrm{g} / \mathrm{mL})$ and insulin $(10 \mu \mathrm{g} / \mathrm{mL})$ (Sigma-Aldrich). PCCL3 cells were transiently transfected with the expression vector pMSCVETV6-NTRK3 or pLVX-AGK-BRAF-GFP, as previously

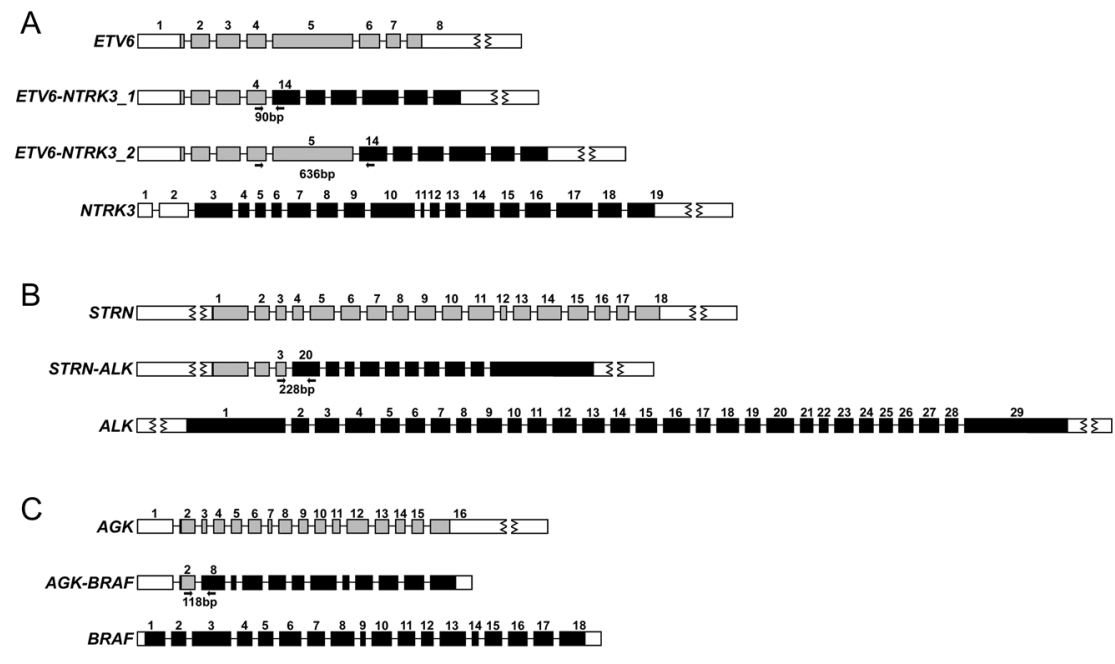

\section{Figure 1}

Diagram of exon/intron structure of ETV6, NTRK3, STRN, ALK, AGK and BRAF genes and the fusion transcripts described in thyroid carcinomas. (A) ETV6 (gray; NM_001987) and NTRK3 (black; NM_002530) genes and the fusion transcripts of ETV6-NTRK3. ETV6-NTRK3_1 (Cosmic ID: COSF1535) and ETV6-NTRK3_2 (Cosmic ID: COSF1537) isoforms correspond to the fusion of exon 4 or exon 5 of ETV6 with exon 14 of NTRK3, respectively. (B) STRN (gray; NM_003162) and ALK (black; NM_004304) genes and the STRN-ALK fusion transcript (Cosmic ID: COSF1431). (C) AGK (gray; NM_018238) and BRAF (black; NM_004333) genes and the $A G K--B R A F$ fusion transcript. Full blocks (gray and black) represent the coding region, and empty blocks represent the untranslated region (UTR). The numbers refer to the exons. The arrows indicate the position of the primers used to amplify all fusion transcripts described in thyroid carcinomas. 
Table 1 PCR primers, annealing temperatures and expected PCR product sizes.

\begin{tabular}{|c|c|}
\hline Gene fusions & Primer sequence $\left(5^{\prime}-3^{\prime}\right)$ \\
\hline ETV6-NTRK $3^{\mathrm{a}}$ & $\begin{array}{l}\text { F: ACACACACAGCCGGAGGTCATAC } \\
\text { R: AGTGGGCTGGCTGAGTCCTCC }\end{array}$ \\
\hline STRN-ALK & $\begin{array}{l}\text { F: GCAACCTTATCCGACTTCTAGC } \\
\text { R: GATACTGGTGCCCGCTCTC }\end{array}$ \\
\hline$A G K-B R A F^{a}$ & $\begin{array}{l}\text { F: CTGCTGACCTGGGGAGGCCATT } \\
\text { R: TCATCTGCTGGTCGGAAGGGCTG }\end{array}$ \\
\hline RPS8 & $\begin{array}{l}\text { F: AACAAGAAATACCGTGCCC } \\
\text { R: GTACGAACCAGCTCGTTATTAG }\end{array}$ \\
\hline
\end{tabular}

\begin{tabular}{c}
\hline Temp $\left({ }^{\circ} \mathrm{C}\right)$ \\
\hline 60 \\
61 \\
60 \\
60
\end{tabular}

\begin{tabular}{lcc}
\hline Location & & Expected product size $(\mathrm{bp})$ \\
\cline { 1 - 1 } Exon 4 & $90 / 636^{\mathrm{b}}$ \\
Exon 14 & 228 \\
Exon 3 & \\
Exon 20 & 118 \\
Exon 2 & \\
Exon 8 & 104 \\
Exon 3 & \\
Exon 4 & \\
\hline
\end{tabular}

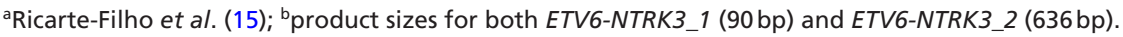

described (20). ETV6-NTRK3 and AGK-BRAF plasmids were kindly donated by Dr James Fagin (Memorial SloanKettering Cancer Center). The oncogene-transfected cells were harvested, and the total RNA was isolated using TRIzol Reagents (Invitrogen) and reverse-transcribed into cDNA using oligo(dT) $)_{12-18}$, as aforementioned. The cDNA generated from cells expressing the fusion transcripts was used as a positive control. For the $S T R N-A L K$ detection, an aliquot of cDNA from a PTC sample known to be positive for $S T R N-A L K$ was used as positive control.

\section{Statistical analyses}

The association between the tumor size and the type of genetic alteration was determined using one-way ANOVA test followed by Bonferroni's post hoc test. To determine the association between the type of genetic alteration and the clinical-pathological features, Fisher's exact test was used with the raw data, and the percentage was shown in the graphics. Statistical analyses were performed using GraphPad Prism v5.01 Software (GraphPad Software).

\section{Results}

\section{ETV6-NTRK3 was exclusively found in follicular variants of PTC}

As two isoforms of ETV6-NTRK3 were previously identified in PTC (13), the set of primers used in this study were designed to detect both isoforms (Fig. 1 and Table 1). Overall, the ETV6-NTRK3 fusion transcript was found in $5 \%(n=6)$ of PTC cases. All positive cases harbor an in-frame fusion of exon 4 of ETV6 to exon 14 of NTRK3 (Fig. 2A and Table 2). Remarkably, ETV6-NTRK3 was exclusively found in FVPTC, leading to a prevalence of about $13 \%(6 / 45)$ in this variant (Table 3). Essentially, $50 \%$ were infiltrative follicular variants of papillary thyroid carcinoma (IFVPTC) and 50\% were encapsulated follicular variants of papillary thyroid carcinoma (EFVPTC) (Table 3). One IFVPTC patient, a 23-year-old female, had lymph node metastasis tissue available and, therefore, it was also screened for the presence of ETV6NTRK3 transcript. The metastatic lymph node tested was also positive for the ETV6-NTRK3 fusion transcript (Fig. 2B). Although non-invasive EFVPTC have very low risk of adverse outcome and were recently reclassified as non-invasive follicular thyroid neoplasia with papillarylike nuclear features (NIFTP), no additional tumor slides and blocks were available to further re-evaluate whether tumors are either NIFTP or invasive EFVPTC.

\section{STRN-ALK was detected in about $3 \%$ of PTC samples}

The STRN-ALK fusion transcript was found in about 3\% $(n=4)$ of PTC samples (Fig. 2C, Tables 2 and 3). All the fusions found were in accordance with the breakpoint already described, corresponding to the in-frame fusion of exon 3 of STRN with exon 20 of ALK (Fig. 2C). The fusion was found in both classical and follicular variants of PTC (Table 3).

\section{No AGK-BRAF was detected in this series of PTC}

Samples were also examined for $A G K-B R A F$ fusion transcript. No PTC case was found to be positive for $A G K-$ $B R A F$ (data not shown), suggesting that $A G K-B R A F$ fusion is mainly associated with pediatric PTC.

\section{ETV6-NTRK3, STRN-ALK and AGK-BRAF are restricted to PTC}

Since most of the fusions were found in FVPTC (78\%; $7 / 9$ ) and as its molecular profile seems to be closer to the FTA/FTC group, the prevalence of ETV6-NTRK3, STRN$A L K$ and $A G K-B R A F$ was also investigated in a series of FTA $(n=22)$ and FTC $(n=19)$. The evaluated FTC and FTA 
A
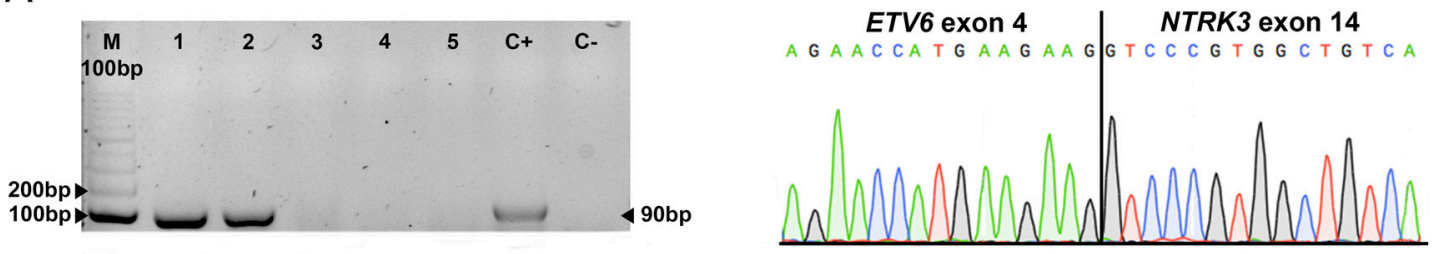

B
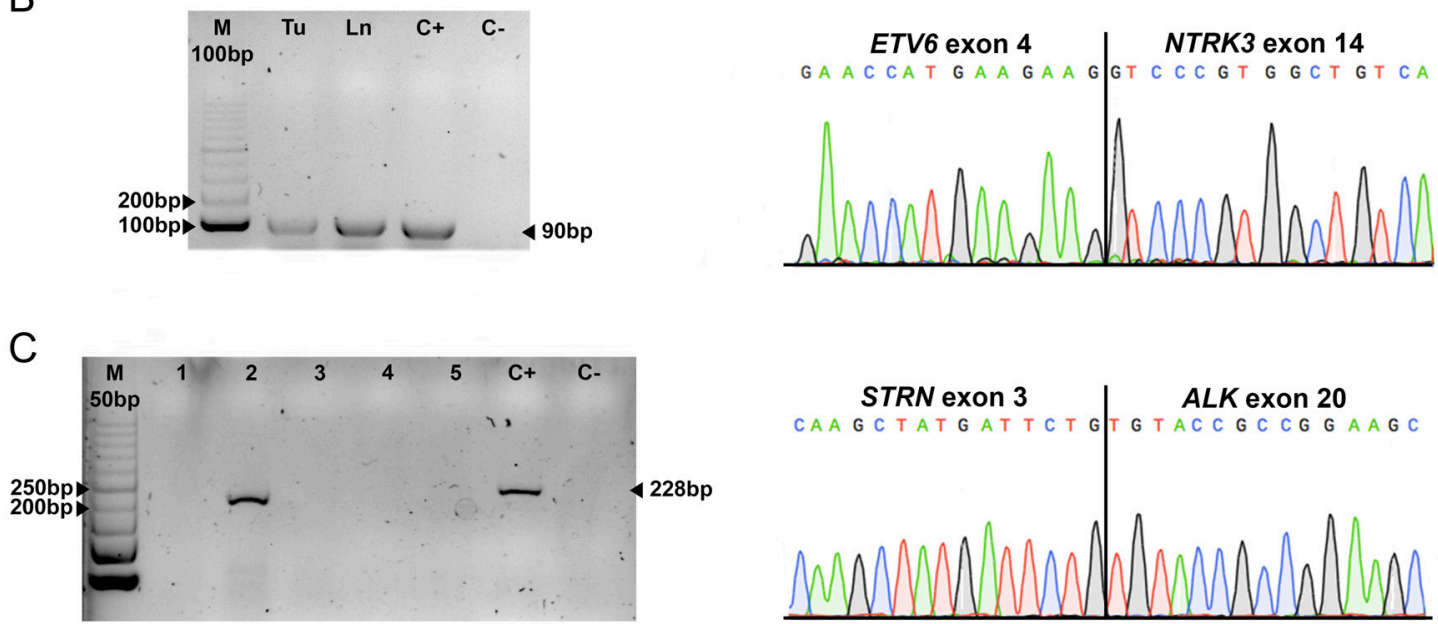

\section{Figure 2}

Fusion transcripts detected in sporadic PTCs. (A) ETV6-NTRK3 fusion transcript identified in sporadic PTCs by standard RT-PCR. Positive (lanes 1 and 2) and negative (lanes 3, 4 and 5) PTC samples are shown. (B) ETV6-NTRK3 fusion transcript in a primary metastatic tumor (Tu) and its matched lymph node metastasis (Ln). (C) STRN-ALK fusion transcript in a sample positive (lane 2) and sample negative (lanes 1, 3, 4 and 5). PCR products submitted to Sanger sequencing to confirm the presence of the fusions. Positive $\left(C_{+}\right)$and negative controls $\left(C_{-}\right)$were included in each run.

samples were negative for these 3 fusions, endorsing that they are specifically associated with PTC subtype.

\section{Gene fusions are found in younger patients and are not associated with aggressive characteristics}

When samples were divided based on the presence of ETV6NTRK3 or STRN-ALK, there was no statistically significant association between with clinico-pathological parameters and the presence of NTRK 3 or $A L K$ fusions. When samples with ETV6-NTRK3 and STRN-ALK fusions were pooled together with those samples who harbor RET/PTC fusions (21) and compared with samples harboring BRAF V600E (19) or NRAS Q61 (21) point mutations or samples that were tested negative for fusions and mutations, we found that fusions were more common in younger patients $(<45$ years old; mean age $36.8 \pm 10.0)$ than mutations $(<45$ years old; mean age: $41.7 \pm 12.3)$. The difference was statistically significant when compared to samples that bear neither mutations nor fusions $(<45$ years old; mean: $52.2 \pm 14.1 ; P<0.01$ ) (Fig. $3 \mathrm{~A}$ and B). Samples harboring point mutations have a more aggressive behavior than samples negative for mutations (Fig. 3C).

\section{Discussion}

The comprehensive analysis of the mutational landscape of PTC $(12,22)$ has provided insights into the molecular alterations that drive its carcinogenesis. Transcriptome sequencing has been particularly useful in helping to identify novel fusions. However, an important question concerns the clinical impact of the found fusions: Are these novel fusions recurrent across multiple patients, tumor subtypes and populations and, therefore, provide the chance to improve the preoperative diagnosis of thyroid nodules and risk stratification?

In this study, we found that the novel fusions ETV6NTRK3 and STRN-ALK (12) are recurrent in this series of PTC from a different geographic area, while they are not 
Table 2 Prevalence of fusion in radiation-induced and sporadic PTC.

\begin{tabular}{l}
\hline Reference \\
\hline Radiation-induced PTC \\
$(15)^{\mathrm{a}}$ \\
$(17)^{\mathrm{b}}$ \\
$(31)^{\mathrm{c}}$ \\
Total $_{\text {Sporadic PTC }}$ \\
Pediatric \\
$(15)^{\mathrm{a}}$ \\
$(16)^{\mathrm{a}}$ \\
$(32)^{\mathrm{a}}$ \\
$(33)^{\mathrm{a}}$ \\
$(28)^{\mathrm{d}}$ \\
Adult \\
$(34)$ \\
$(17)$ \\
$(14)$ \\
$(13)$ \\
$(12)$ \\
$(21)$ \\
$(28)^{\mathrm{d}}$ \\
This study \\
Adult and pediatric \\
$(27)$ \\
Total \\
\hline
\end{tabular}

\begin{tabular}{c}
\hline Samples \\
\hline 26 \\
62 \\
67 \\
155
\end{tabular}

\begin{tabular}{c}
\hline ETV6-NTRK3, $n(\%)$ \\
\hline $2(8 \%)$ \\
$9(14 \%)$ \\
$4(6 \%)$ \\
$15 / 155(10 \%)$
\end{tabular}

\begin{tabular}{c}
\hline STRN-ALK, $n$ (\%) \\
\hline NE \\
NE \\
NE \\
NE
\end{tabular}

\begin{tabular}{c}
\hline AGK-BRAF, $n$ (\%) \\
\hline $1(4 \%)$ \\
$\mathrm{NE}$ \\
$\mathrm{NE}$ \\
$1 / 26(4 \%)$
\end{tabular}

Studies performed in samples of pediatric patients with $<18$ years; ${ }^{b}$ study performed in patients who were aged $<18$ years at the time of Chernobyl accident; 'study performed only with young adult patient samples ( $<22$ years old); ${ }^{\text {in }}$ this study, only patients $<40$ years old $(n=41)$ were further elucidated. The data shown in this table are only from these patients. No data about fusion partners from patients $>40$ years old ( $n=303$ ) were available

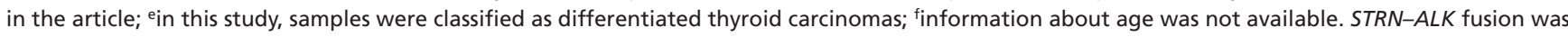
observed in one pediatric patient (13 years old) and in one adult patient (50 years old).

$\mathrm{NE}$, not evaluated.

present in benign lesions and, therefore, may have an important impact on the diagnosis of thyroid nodules in adult population from different populations. AGK-BRAF, on the other hand, is not found in adult population and could have an important impact only in PTC from pediatric population. Whether they might have impact on the prognosis, it is still not clear.

Regarding NTRK3 fusions, although several 5' partners were detected in PTC, the majority of them were present in only one sample (12). Interestingly, the ETV6-NTRK3

Table 3 The demographic and clinico-pathological features of PTCs positive for ETV6-NTRK3 and STRN-ALK fusions.

\begin{tabular}{l}
\hline ID \\
\hline ETV6-NTRK3 \\
$1^{\mathrm{a}}$ \\
2 \\
3 \\
4 \\
5 \\
6 \\
STRN-ALK \\
$1^{\mathrm{a}}$ \\
7 \\
8 \\
9
\end{tabular}

\begin{tabular}{|c|c|}
\hline Sex & Age (years) \\
\hline$F$ & 43 \\
\hline $\mathrm{M}$ & 23 \\
\hline$F$ & 31 \\
\hline$F$ & 48 \\
\hline $\mathrm{F}$ & 42 \\
\hline $\mathrm{F}$ & NA \\
\hline $\mathrm{F}$ & 43 \\
\hline $\mathrm{F}$ & 43 \\
\hline $\mathrm{F}$ & 47 \\
\hline M & 34 \\
\hline
\end{tabular}

\begin{tabular}{c}
\hline Size $(\mathrm{cm})$ \\
\hline \\
2.5 \\
2.5 \\
NA \\
2.8 \\
2.0 \\
3.0 \\
\\
2.5 \\
3.5 \\
1.5 \\
2.2 \\
\hline
\end{tabular}

\begin{tabular}{l} 
Histological variant \\
\hline IFVPTC \\
IFVPTC \\
EFVPTC \\
EFVPTC \\
IFVPTC \\
EFVPTC \\
IFVPTC \\
FVPTCC \\
Classical \\
Classical
\end{tabular}

\begin{tabular}{c}
\hline Multifocality \\
\hline No \\
Yes \\
No \\
No \\
Yes \\
No \\
No \\
No \\
Yes \\
Yes
\end{tabular}

\begin{tabular}{l} 
ETE \\
\hline No \\
Yes \\
NA \\
No \\
Yes \\
No \\
No \\
No \\
Yes \\
No \\
\hline
\end{tabular}

\begin{tabular}{cccc} 
LNM & & Recurrence \\
\cline { 1 - 1 } No & & No \\
Yes & & & Yes \\
No & & No \\
No & & No \\
No & & NA \\
No & & No \\
& & \\
No & & No \\
No & & No \\
No & & No \\
Yes & & No
\end{tabular}

aPatient 1 was positive for both ETV6-NTRK3 and STRN-ALK, and also for RETIPTC2 (30); blymph node metastasis from patient 2 was also positive for ETV6-NTRK3; information regarding the presence/absence of capsule is not available.

ETE, extrathyroidal extension; LNM, lymph node metastasis; F, female; M, male; NA, not available; IFVPTC, infiltrative (non-encapsulated) follicular variant of papillary thyroid carcinoma; EFVPTC, encapsulated follicular variant of papillary thyroid carcinoma. 

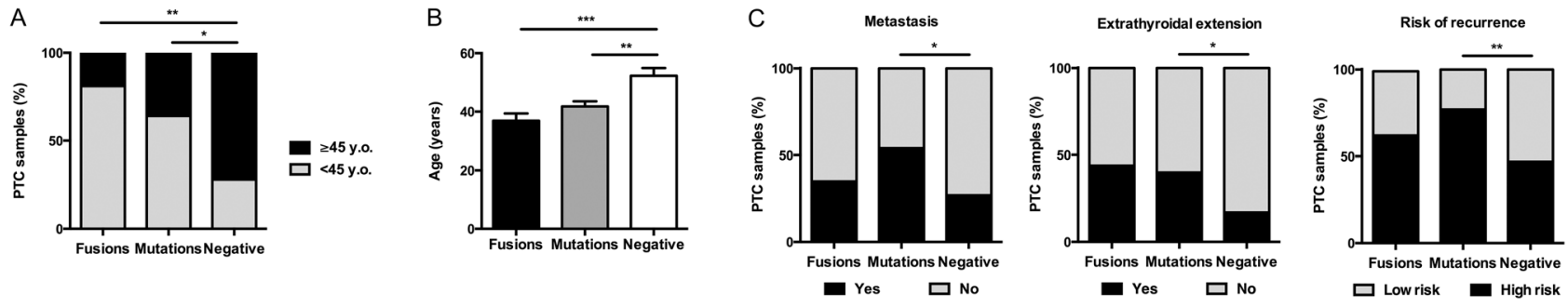

\section{Figure 3}

PTC samples divided into 3 groups according to the mutational status. Fusions: PTC samples with ETV6-NTRK3, STRN-ALK and RETIPTC (21) fusions. Mutations: PTC samples with point mutations in BRAF gene (19) or NRAS (21). Negative: those who bear neither fusions nor mutations. (A) Patients were classified according to age ( $\leq 45$ vs $>45$ years old). Fusions were more prevalent in younger patients than negative. (B) Mean age of diagnosis of patients harboring fusions (age 36.8 \pm 10.0 ), mutations $(41.7 \pm 12.3$ ) or negative (mean: $52.2 \pm 14.1$ ). (C) Percentage of patients with the presence of metastasis, extrathyroidal extension and risk of recurrence in each group (fusion, mutation and negative). ${ }^{*} P<0.05 ; * * P<0.01$.

fusion was identified as a common event in PTCs from patients who were exposed to radiation (15), and was also reported in sporadic pediatric (13) and adult PTCs (15). Afterward, it has been confirmed that ETV6-NTRK3 is a recurrent event in PTC $(12,22)$ and is also able to induce MAPK activation and promote cell growth and transformation (13). Two ETV6-NTRK3 isoforms have been identified in thyroid samples (15). Most of the fusions reported involve exon 4 of ETV6 and exon 14 of NTRK3, but one case involved fusion of exon 5 of ETV6 and exon 14 of NTRK3 (13). Therefore, we investigated the prevalence of ETV6-NTRK3 fusion in a large set of sporadic PTCs, using a set of primers that would allow us to detect both isoforms. We here identified ETV6-NTRK3 in six PTC cases. The prevalence (5\%) is slightly higher than previously reported in adults but still lower than that reported in radiation-exposed PTC. Remarkably, all ETV6-NTRK3-positive cases were from FVPTC.

FVPTC is recognized as a tumor composed of follicles rather than papillae, but with cells presenting the nuclear features of PTC, and can be further divided into infiltrative (IFVPTC) or encapsulated (EFVPTC). It has been suggested that infiltrative tumors were more likely to have extrathyroidal extension and lymph node metastases, and its biological behaviors are similar to the conventional PTC, while the encapsulated behaves more like follicular thyroid tumors. At the molecular levels, it has been suggested that the non-invasive EFVPTC have a high prevalence of mutations that are usually associated with follicular-patterned thyroid tumors such as FTA and FTC $(22,23,24)$. As we found ETV6-NTRK3 fusion in three EFVPTC, we tested whether other follicular-patterned lesions, such as the benign FTAs and the malignant FTCs, could harbor ETV6-NTRK3 fusion. None of the benign
FTAs or malignant FTCs were positive for ETV6-NTRK3. Therefore, ETV6-NTRK3 fusion has the potential to serve as a diagnostic marker for FVPTC. Since FVPTC represents a diagnostic challenge on fine-needle aspiration cytology and the percentage of follicular-patterned thyroid malignancies that proved to be FVPTC on final biopsy has increased, these findings have important clinical implications.

Regarding the prognostic implications, most ETV6NTRK3-positive cases were under the age of 45 years, two cases had extrathyroidal extension and one case had lymph node metastasis. When both the paired primary tumor and the lymph node metastases were profiled, the ETV6-NTRK3 fusion was also found in the lymph node metastases. The fact that this fusion was found in IFVPTC, as well as in the paired lymph node metastases, raises the possibility that tumors with ETV6-NTRK3 fusion might have the potential to metastasize. However, whether additional mutations are needed to tumor progression, we still do not know.

Although $A L K$ fusions are rare in thyroid cancer, aberrant ALK activation due to rearrangements leads to constitutive activation of the MAPK pathway in both PTC (16) and other tumor subtypes $(25,26)$. While originally found in PTC with more advanced stage of disease and tumors prone to dedifferentiation (16), STKN-ALK fusion has also been described in classical PTC $(17,22)$. Although other ALK fusion was described in medullary thyroid carcinoma (MTC) (27), STKN-ALK fusion was not found in FTC and MTC (16). In our cohort, four PTCs exhibited the $S T R N-A L K$ fusion. Although no association was found with demographic and clinico-pathological features such as histological subtypes, age and aggressiveness, one of the four PTC samples positive for STRN-ALK had lymph 
node metastasis at diagnosis. Interestingly, the STRN$A L K$ fusions found in pediatric PTCs were metastatic or of a solid variant, indicating its association with a more aggressive phenotype even in pediatric samples $(28,29)$.

Importantly, the ALK inhibitor, crizotinib, has been shown to be effective in the treatment of patients with thyroid tumors harboring STRN-ALK (30) or other ALK fusions (27). A more detailed understanding of the downstream targets these fusions affect and the cellular process they are involved may help to find novel therapeutic targets.

Although common, a wide range of different $5^{\prime}$ partners were found to be fused in-frame to $B R A F$, and most were non-recurrent events (12). AGK-BRAF was found as a recurrent event in sporadic pediatric PTC samples (14) and in one radiation-exposed PTC case (13); however, in the TCGA cohort, only one PTC exhibited $A G K-B R A F$ fusion. No $A G K-B R A F$ fusion was found in over 100 adult-sporadic PTCs, endorsing that $A G K-B R A F$ is an event likely associated with PTCs of younger age, while BRAF V600E is the leading genetic event in adultsporadic PTC $(12,22)$.

Finally, nearly 26\% (30/116) of all PTC samples in this cohort are negative for the most prevalent events described in PTC $(19,21)$.

The integrated view of RET/PTC fusions previously reported in this cohort (21), and those reported here, supports that these kinase fusions are recurrent events in PTC. Additionally, fusions were more prevalent in younger patients ( $<45$ years old), while point mutations were associated with a more aggressive tumor phenotype. The identification of these fusions in thyroid carcinomas not only helps to expand our knowledge about the landscape of PTC but it also suggests that ETV6-NTRK3 and $S T R N-A L K$ are useful for screening and therapeutic targets. Further analysis is still needed to delineate the comprehensive panorama of PTC.

\section{Declaration of interest}

The authors declare that there is no conflict of interest that could be perceived as prejudicing the impartiality of this study.

Funding

The study was supported by research grants from The São Paulo State Research Foundation (FAPESP), grant numbers 2013/03867-5 and 2015/60330-8, and from The Brazilian Research Council (CNPq), grant number 470441/2013-5. AUB was a recipient of fellowship from FAPESP (2012/06221-6). J M C is a recipient of a scholarship of Research Productivity from CNPq.

\section{Acknowledgements}

The authors thank Prof. James A Fagin (Memorial Sloan-Kettering Cancer Center, New York, NY, USA) for providing the plasmids encoding ETV6$N T R K 3$ and $A G K-B R A F$ fusions.

\section{References}

1 Mitelman F, Johansson B \& Mertens F. The impact of translocations and gene fusions on cancer causation. Nature Reviews Cancer 20077 233-245. (https://doi.org/10.1038/nrc2091)

2 Mertens F, Johansson B, Fioretos T \& Mitelman F. The emerging complexity of gene fusions in cancer. Nature Reviews Cancer 201515 371-381. (https://doi.org/10.1038/nrc3947)

3 Fusco A, Grieco M, Santoro M, Berlingieri MT, Pilotti S, Pierotti MA, Della Porta G \& Vecchio G. A new oncogene in human thyroid papillary carcinomas and their lymph-nodal metastases. Nature 1987 328 170-172. (https://doi.org/10.1038/328170a0)

4 Grieco M, Santoro M, Berlingieri MT, Melillo RM, Donghi R, Bongarzone I, Pierotti MA, Della Porta G, Fusco A \& Vecchio G. PTC is a novel rearranged form of the ret proto-oncogene and is frequently detected in vivo in human thyroid papillary carcinomas. Cell 199060 557-563. (https://doi.org/10.1016/00928674(90)90659-3)

5 Pierotti MA, Santoro M, Jenkins RB, Sozzi G, Bongarzone I, Grieco M, Monzini N, Miozzo M, Herrmann MA, Fusco A et al. Characterization of an inversion on the long arm of chromosome 10 juxtaposing d10s170 and ret and creating the oncogenic sequence ret/PTC. PNAS 199289 1616-1620. (https://doi.org/10.1073/ pnas.89.5.1616)

6 Menicali E, Moretti S, Voce P, Romagnoli S, Avenia N \& Puxeddu E. Intracellular signal transduction and modification of the tumor microenvironment induced by ret/PTCs in papillary thyroid carcinoma. Frontiers in Endocrinology 20123 67. (https://doi. org/10.3389/fendo.2012.00067)

7 Xing M. Molecular pathogenesis and mechanisms of thyroid cancer. Nature Reviews Cancer 201313 184-199. (https://doi.org/10.1038/ nrc3431)

8 Romei C, Ciampi R \& Elisei R. A comprehensive overview of the role of the ret proto-oncogene in thyroid carcinoma. Nature Reviews Endocrinology 201612 192-202. (https://doi.org/10.1038/ nrendo.2016.11)

9 Santoro M, Chiappetta G, Cerrato A, Salvatore D, Zhang L, Manzo G, Picone A, Portella G, Santelli G, Vecchio G et al. Development of thyroid papillary carcinomas secondary to tissue-specific expression of the ret/PTC1 oncogene in transgenic mice. Oncogene 199612 1821-1826.

10 Jhiang SM, Sagartz JE, Tong Q, Parker-Thornburg J, Capen CC, Cho JY, Xing S \& Ledent C. Targeted expression of the ret/PTC1 oncogene induces papillary thyroid carcinomas. Endocrinology 1996 137 375-378. (https://doi.org/10.1210/endo.137.1.8536638)

11 Powell DJ Jr, Russell J, Nibu K, Li G, Rhee E, Liao M, Goldstein M, Keane WM, Santoro M, Fusco Aet al. The ret/PTC3 oncogene: metastatic solid-type papillary carcinomas in murine thyroids. Cancer Research 199858 5523-5528.

12 Cancer Genome Atlas Research Network. Integrated genomic characterization of papillary thyroid carcinoma. Cell 2014159 676-690. (https://doi.org/10.1016/j.cell.2014.09.050)

13 Perot G, Soubeyran I, Ribeiro A, Bonhomme B, Savagner F, BoutetBouzamondo N, Hostein I, Bonichon F, Godbert Y \& Chibon F. Identification of a recurrent STRN/ALK fusion in thyroid carcinomas. PLoS ONE 20149 e87170. (https://doi.org/10.1371/journal. pone.0087170) 
14 Kelly LM, Barila G, Liu P, Evdokimova VN, Trivedi S, Panebianco F, Gandhi M, Carty SE, Hodak SP, Luo J et al. Identification of the transforming STRN-ALK fusion as a potential therapeutic target in the aggressive forms of thyroid cancer. PNAS $20141114233-4238$. (https://doi.org/10.1073/pnas.1321937111)

15 Ricarte-Filho JC, Li S, Garcia-Rendueles ME, Montero-Conde C, Voza F, Knauf JA, Heguy A, Viale A, Bogdanova T, Thomas GA et al. Identification of kinase fusion oncogenes in post-chernobyl radiation-induced thyroid cancers. Journal of Clinical Investigation 2013123 4935-4944. (https://doi.org/10.1172/JCI69766)

16 Leeman-Neill RJ, Kelly LM, Liu P, Brenner AV, Little MP, Bogdanova TI, Evdokimova VN, Hatch M, Zurnadzy LY, Nikiforova MN et al. ETV6-NTRK3 is a common chromosomal rearrangement in radiation-associated thyroid cancer. Cancer 2014 120 799-807. (https://doi.org/10.1002/cncr.28484)

17 Cordioli MI, Moraes L, Carvalheira G, Sisdelli L, Alves MT, Delcelo R, Monte O, Longui CA, Cury AN \& Cerutti JM. AGKBRAF gene fusion is a recurrent event in sporadic pediatric thyroid carcinoma. Cancer Medicine 20165 1535-1541. (https://doi. org/10.1002/cam4.698)

18 Cerutti JM, Latini FR, Nakabashi C, Delcelo R, Andrade VP, Amadei MJ, Maciel RM, Hojaij FC, Hollis D, Shoemaker J et al. Diagnosis of suspicious thyroid nodules using four protein biomarkers. Clinical Cancer Research 200612 3311-3318. (https://doi. org/10.1158/1078-0432.CCR-05-2226)

19 Oler G \& Cerutti JM. High prevalence of BRAF mutation in a Brazilian cohort of patients with sporadic papillary thyroid carcinomas: correlation with more aggressive phenotype and decreased expression of iodide-metabolizing genes. Cancer 2009115 972-980. (https://doi.org/10.1002/cncr.24118)

20 Oliveira MN, Hemerly JP, Bastos AU, Tamanaha R, Latini FR, Camacho CP, Impellizzeri A, Maciel RM \& Cerutti JM. The ret p.G533c mutation confers predisposition to multiple endocrine neoplasia type $2 \mathrm{a}$ in a Brazilian kindred and is able to induce a malignant phenotype in vitro and in vivo. Thyroid 201121 975-985. (https://doi.org/10.1089/thy.2010.0190)

21 Bastos AU, Oler G, Nozima BH, Moyses RA \& Cerutti JM. BRAF v600e and decreased NIS and TPO expression are associated with aggressiveness of a subgroup of papillary thyroid microcarcinoma. European Journal of Endocrinology 2015173 525-540. (https://doi. org/10.1530/EJE-15-0254)

22 Yoo SK, Lee S, Kim SJ, Jee HG, Kim BA, Cho H, Song YS, Cho SW, Won JK, Shin JY et al. Comprehensive analysis of the transcriptional and mutational landscape of follicular and papillary thyroid cancers. PLoS Genetics 201612 e1006239. (https://doi.org/10.1371/journal. pgen.1006239)

23 Seethala RR, Chiosea SI, Liu CZ, Nikiforova M \& Nikiforov YE. Clinical and morphologic features of ETV6-NTRK3 translocated papillary thyroid carcinoma in an adult population without radiation exposure. American Journal of Surgical Pathology 201741 446-457. (https://doi.org/10.1097/PAS.0000000000000814)

24 Nikiforov YE, Seethala RR, Tallini G, Baloch ZW, Basolo F, Thompson LD, Barletta JA, Wenig BM, Al Ghuzlan A, Kakudo K et al. Nomenclature revision for encapsulated follicular variant of papillary thyroid carcinoma: a paradigm shift to reduce overtreatment of indolent tumors. JAMA Oncology 20162 1023-1029. (https://doi. org/10.1001/jamaoncol.2016.0386)

25 Hrustanovic G \& Bivona TG. RAS signaling in ALK fusion lung cancer. Small GTPases 20167 32-33. (https://doi.org/10.1080/215412 48.2015.1131803)

26 Okamoto I \& Nakagawa K. Echinoderm microtubule-associated protein-like 4-anaplastic lymphoma kinase-targeted therapy for advanced non-small cell lung cancer: molecular and clinical aspects. Cancer Science 2012103 1391-1396. (https://doi.org/10.1111/j.13497006.2012.02327.x)

27 Ji JH, Oh YL, Hong M, Yun JW, Lee HW, Kim D, Ji Y, Kim DH, Park WY, Shin HT et al. Identification of driving ALK fusion genes and genomic landscape of medullary thyroid cancer. PLoS Genetics 201511 e1005467. (https://doi.org/10.1371/journal.pgen.1005467)

28 Park G, Kim TH, Lee HO, Lim JA, Won JK, Min HS, Lee KE, Park DJ, Park YJ \& Park WY. Standard immunohistochemistry efficiently screens for anaplastic lymphoma kinase rearrangements in differentiated thyroid cancer. Endocrine-Related Cancer 201522 55-63. (https://doi.org/10.1530/ERC-14-0467)

29 Vanden Borre P, Schrock AB, Anderson PM, Morris JC 3rd, Heilmann AM, Holmes O, Wang K, Johnson A, Waguespack SG, Ou SI et al. Pediatric, adolescent, and young adult thyroid carcinoma harbors frequent and diverse targetable genomic alterations, including kinase fusions. Oncologist 201722 255-263. (https://doi. org/10.1634/theoncologist.2016-0279)

30 Godbert Y, Henriques de Figueiredo B, Bonichon F, Chibon F, Hostein I, Perot G, Dupin C, Daubech A, Belleannee G, Gros A et al. Remarkable response to crizotinib in woman with anaplastic lymphoma kinase-rearranged anaplastic thyroid carcinoma. Journal of Clinical Oncology 201533 e84-e87. (https://doi.org/10.1200/ JCO.2013.49.6596)

31 Mitsutake N, Fukushima T, Matsuse M, Rogounovitch T, Saenko V, Uchino S, Ito M, Suzuki K, Suzuki S \& Yamashita S. BRAF(v600e) mutation is highly prevalent in thyroid carcinomas in the young population in Fukushima: a different oncogenic profile from chernobyl. Scientific Reports 20155 16976. (https://doi.org/10.1038/ srep16976)

32 Picarsic JL, Buryk MA, Ozolek J, Ranganathan S, Monaco SE, Simons JP, Witchel SF, Gurtunca N, Joyce J, Zhong S et al. Molecular characterization of sporadic pediatric thyroid carcinoma with the DNA/RNA thyroseq v2 next-generation sequencing assay. Pediatric and Developmental Pathology 201619 115-122. (https://doi. org/10.2350/15-07-1667-OA.1)

33 Prasad ML, Vyas M, Horne MJ, Virk RK, Morotti R, Liu Z, Tallini G, Nikiforova MN, Christison-Lagay ER, Udelsman R et al. NTRK fusion oncogenes in pediatric papillary thyroid carcinoma in northeast united states. Cancer 2016122 1097-1107. (https://doi. org/10.1002/cncr.29887)

34 Smallridge RC, Chindris AM, Asmann YW, Casler JD, Serie DJ, Reddi HV, Cradic KW, Rivera M, Grebe SK, Necela BM et al. RNA sequencing identifies multiple fusion transcripts, differentially expressed genes, and reduced expression of immune function genes in BRAF (v600e) mutant vs BRAF wild-type papillary thyroid carcinoma. Journal of Clinical Endocrinology and Metabolism 201499 E338-E347. (https://doi.org/10.1210/jc.2013-2792)
Received 23 June 2017

Revised version received 8 October 2017

Accepted 16 October 2017 